\title{
ADDITIVE REVERSES OF THE CONTINUOUS TRIANGLE INEQUALITY FOR BOCHNER INTEGRAL OF VECTOR-VALUED FUNCTIONS IN HILBERT SPACES
}

\author{
SEVER S. DRAGOMIR
}

\begin{abstract}
Some additive reverses of the continuous triangle inequality for Bochner integral of vector-valued functions in Hilbert spaces are given. Applications for complex-valued functions are provided as well.
\end{abstract}

\section{INTRODUCTION}

Let $f:[a, b] \rightarrow \mathbb{K}, \mathbb{K}=\mathbb{C}$ or $\mathbb{R}$ be a Lebesgue integrable function. The following inequality is the continuous version of the triangle inequality

$$
\left|\int_{a}^{b} f(x) d x\right| \leq \int_{a}^{b}|f(x)| d x,
$$

and plays a fundamental role in Mathematical Analysis and its applications.

It seems, see [5. p. 492], that the first reverse inequality for (1.1) was obtained by J. Karamata in his book from 1949, [3] :

$$
\cos \theta \int_{a}^{b}|f(x)| d x \leq\left|\int_{a}^{b} f(x) d x\right|
$$

provided

$$
|\arg f(x)| \leq \theta, \quad x \in[a, b],
$$

where $\theta$ is a given angle in $\left(0, \frac{\pi}{2}\right)$.

This integral inequality is the continuous version of a reverse inequality for the generalised triangle inequality

$$
\cos \theta \sum_{i=1}^{n}\left|z_{i}\right| \leq\left|\sum_{i=1}^{n} z_{i}\right|,
$$

provided

$$
a-\theta \leq \arg \left(z_{i}\right) \leq a+\theta, \text { for } i \in\{1, \ldots, n\},
$$

where $a \in \mathbb{R}$ and $\theta \in\left(0, \frac{\pi}{2}\right)$, which, as pointed out in [5] p. 492], was first discovered by M. Petrovich in 1917, [6], and, subsequently rediscovered by other authors, including J. Karamata [3. p. $300-301]$, H.S. Wilf [7, and in an equivalent form by M. Marden [4.

The first to consider the problem for sums in the general case of Hilbert and Banach spaces, were J.B. Diaz and F.T. Metcalf [1].

Date: April 16, 2004.

2000 Mathematics Subject Classification. 46C05, 26D15, 26D10.

Key words and phrases. Triangle inequality, Reverse inequality, Hilbert spaces, Bochner integral. 
In our previous work [2], we pointed out some continuous versions of Diaz and Metcalf reverses of the generalised triangle inequality.

We mention here some results from [2] which may be compared with the new results obtained in Sections 2 and 3 below.

Theorem 1. If $f \in L([a, b] ; H)$, the space of Bochner integrable functions defined on $[a, b]$ and with values in the Hilbert space $H$, and there exists a constant $K \geq 1$ and a vector $e \in H,\|e\|=1$ such that

$$
\|f(t)\| \leq K \operatorname{Re}\langle f(t), e\rangle \quad \text { for a.e. } t \in[a, b],
$$

then we have the inequality:

$$
\int_{a}^{b}\|f(t)\| d t \leq K\left\|\int_{a}^{b} f(t) d t\right\| .
$$

The case of equality holds in (1.5) if and only if

$$
\int_{a}^{b} f(t) d t=\frac{1}{K}\left(\int_{a}^{b}\|f(t)\| d t\right) \cdot e .
$$

As particular cases of interest that may be applied in practice, we note the following corollaries established in [2].

Corollary 1. Let e be a unit vector in the Hilbert space $(H ;\langle\cdot, \cdot\rangle), \rho \in(0,1)$ and $f \in L([a, b] ; H)$ so that

$$
\|f(t)-e\| \leq \rho \text { for a.e. } t \in[a, b] .
$$

Then we have the inequality

$$
\sqrt{1-\rho^{2}} \int_{a}^{b}\|f(t)\| d t \leq\left\|\int_{a}^{b} f(t) d t\right\|
$$

with equality if and only if

$$
\int_{a}^{b} f(t) d t=\sqrt{1-\rho^{2}}\left(\int_{a}^{b}\|f(t)\| d t\right) \cdot e .
$$

Corollary 2. Let e be a unit vector in $H$ and $M \geq m>0$. If $f \in L([a, b] ; H)$ is such that

$$
\operatorname{Re}\langle M e-f(t), f(t)-m e\rangle \geq 0 \text { for a.e. } t \in[a, b],
$$

or, equivalently,

$$
\left\|f(t)-\frac{M+m}{2} e\right\| \leq \frac{1}{2}(M-m) \quad \text { for a.e. } t \in[a, b],
$$

then we have the inequality

$$
\frac{2 \sqrt{m M}}{M+m} \int_{a}^{b}\|f(t)\| d t \leq\left\|\int_{a}^{b} f(t) d t\right\|
$$

or, equivalently,

$$
0 \leq \int_{a}^{b}\|f(t)\| d t-\left\|\int_{a}^{b} f(t) d t\right\| \leq \frac{(\sqrt{M}-\sqrt{m})^{2}}{M+m}\left\|\int_{a}^{b} f(t) d t\right\| .
$$


The equality holds in 2.11) (or in the second part of (2.12) if and only if

$$
\int_{a}^{b} f(t) d t=\frac{2 \sqrt{m M}}{M+m}\left(\int_{a}^{b}\|f(t)\| d t\right) e .
$$

The main aim of this paper is to point out additive reverses for the continuous triangle inequality, namely, upper bounds for the nonnegative difference

$$
\int_{a}^{b}\|f(t)\| d t-\left\|\int_{a}^{b} f(t) d t\right\|
$$

under various assumptions on the function $f \in L([a, b] ; H)$.

Both the case for a unit vector $e \in H$ and a family of orthonormal vectors $\left\{e_{i}\right\}_{i \in\{1, \ldots, n\}}$ are analysed. Applications for complex-valued Lebesgue integrable functions are given as well.

\section{Some Additive Reverses for a Unit Vector}

The following result holds.

Theorem 2. If $f \in L([a, b] ; H)$ is such that there exists a vector $e \in H,\|e\|=1$ and $k:[a, b] \rightarrow[0, \infty)$, a Lebesgue integrable function with

$$
\|f(t)\|-\operatorname{Re}\langle f(t), e\rangle \leq k(t) \quad \text { for a.e. } t \in[a, b],
$$

then we have the inequality:

$$
(0 \leq) \int_{a}^{b}\|f(t)\| d t-\left\|\int_{a}^{b} f(t) d t\right\| \leq \int_{a}^{b} k(t) d t .
$$

The equality holds in (2.2) if and only if

$$
\int_{a}^{b}\|f(t)\| d t \geq \int_{a}^{b} k(t) d t
$$

and

$$
\int_{a}^{b} f(t) d t=\left(\int_{a}^{b}\|f(t)\| d t-\int_{a}^{b} k(t) d t\right) e .
$$

Proof. If we integrate the inequality (2.1), we get

$$
\int_{a}^{b}\|f(t)\| d t \leq \operatorname{Re}\left\langle\int_{a}^{b} f(t) d t, e\right\rangle+\int_{a}^{b} k(t) d t .
$$

By Schwarz's inequality for $e$ and $\int_{a}^{b} f(t) d t$, we have

$$
\begin{aligned}
\operatorname{Re}\left\langle\int_{a}^{b} f(t) d t, e\right\rangle & \leq\left|\operatorname{Re}\left\langle\int_{a}^{b} f(t) d t, e\right\rangle\right| \leq\left|\left\langle\int_{a}^{b} f(t) d t, e\right\rangle\right| \\
& \leq\left\|\int_{a}^{b} f(t) d t\right\|\|e\|=\left\|\int_{a}^{b} f(t) d t\right\| .
\end{aligned}
$$

Making use of (2.5) and (2.6), we deduce the desired inequality (2.2). 
If (2.3) and (2.4) hold true, then

$$
\left\|\int_{a}^{b} f(t) d t\right\|=\left|\int_{a}^{b}\|f(t)\| d t-\int_{a}^{b} k(t) d t\right|\|e\|=\int_{a}^{b}\|f(t)\| d t-\int_{a}^{b} k(t) d t
$$

and the equality holds true in (2.2).

Conversely, if the equality holds in (2.2), then, obviously (2.3) is valid and we need only to prove (2.4).

If $\|f(t)\|-\operatorname{Re}\langle f(t), e\rangle<k(t)$ for a.e. $t \in[a, b]$, then (2.5) holds as a strict inequality, implying that (2.2) also holds as a strict inequality. Therefore, if we assume that equality holds in (2.2), then we must have

$$
\|f(t)\|=\operatorname{Re}\langle f(t), e\rangle+k(t) \text { for a.e. } t \in[a, b] .
$$

It is well known that in Schwarz's inequality $\|x\|\|y\| \geq \operatorname{Re}\langle x, y\rangle$ the equality holds iff there exists a $\lambda \geq 0$ such that $x=\lambda y$. Therefore, if we assume that the equality holds in all of (2.6), then there exists a $\lambda \geq 0$ such that

$$
\int_{a}^{b} f(t) d t=\lambda e
$$

Integrating (2.7) on $[a, b]$, we deduce

$$
\int_{a}^{b}\|f(t)\| d t=\operatorname{Re}\left\langle\int_{a}^{b} f(t) d t, e\right\rangle+\int_{a}^{b} k(t) d t
$$

and thus, by (2.8), we get

$$
\int_{a}^{b}\|f(t)\| d t=\lambda\|e\|^{2}+\int_{a}^{b} k(t) d t
$$

giving $\lambda=\int_{a}^{b}\|f(t)\| d t-\int_{a}^{b} k(t) d t$.

Using (2.8), we deduce (2.4) and the theorem is completely proved.

The following corollary may be useful for applications.

Corollary 3. If $f \in L([a, b] ; H)$ is such that there exists a vector $e \in H,\|e\|=1$ and $\rho \in(0,1)$ such that

$$
\|f(t)-e\| \leq \rho \quad \text { for a.e. } t \in[a, b],
$$

then we have the inequality

$$
\begin{aligned}
& (0 \leq) \int_{a}^{b}\|f(t)\| d t-\left\|\int_{a}^{b} f(t) d t\right\| \\
& \leq \frac{\rho^{2}}{\sqrt{1-\rho^{2}}\left(1+\sqrt{1-\rho^{2}}\right)} \operatorname{Re}\left\langle\int_{a}^{b} f(t) d t, e\right\rangle \\
& \left(\leq \frac{\rho^{2}}{\sqrt{1-\rho^{2}}\left(1+\sqrt{1-\rho^{2}}\right)}\left\|\int_{a}^{b} f(t) d t\right\|\right) .
\end{aligned}
$$

The equality holds in 2.10) if and only if

$$
\int_{a}^{b}\|f(t)\| d t \geq \frac{\rho^{2}}{\sqrt{1-\rho^{2}}\left(1+\sqrt{1-\rho^{2}}\right)} \operatorname{Re}\left\langle\int_{a}^{b} f(t) d t, e\right\rangle
$$


and

$$
\begin{array}{rl}
\int_{a}^{b} & f(t) d t \\
& =\left(\int_{a}^{b}\|f(t)\| d t-\frac{\rho^{2}}{\sqrt{1-\rho^{2}}\left(1+\sqrt{1-\rho^{2}}\right)} \operatorname{Re}\left\langle\int_{a}^{b} f(t) d t, e\right\rangle\right) e .
\end{array}
$$

Proof. Firstly, note that (2.3) is equivalent to

$$
\|f(t)\|^{2}+1-\rho^{2} \leq 2 \operatorname{Re}\langle f(t), e\rangle,
$$

giving

$$
\frac{\|f(t)\|^{2}}{\sqrt{1-\rho^{2}}}+\sqrt{1-\rho^{2}} \leq \frac{2 \operatorname{Re}\langle f(t), e\rangle}{\sqrt{1-\rho^{2}}}
$$

for a.e. $t \in[a, b]$.

Since, obviously

$$
2\|f(t)\| \leq \frac{\|f(t)\|^{2}}{\sqrt{1-\rho^{2}}}+\sqrt{1-\rho^{2}}
$$

for any $t \in[a, b]$, then we deduce the inequality

$$
\|f(t)\| \leq \frac{\operatorname{Re}\langle f(t), e\rangle}{\sqrt{1-\rho^{2}}} \text { for a.e. } t \in[a, b],
$$

which is clearly equivalent to

$$
\|f(t)\|-\operatorname{Re}\langle f(t), e\rangle \leq \frac{\rho^{2}}{\sqrt{1-\rho^{2}}\left(1+\sqrt{1-\rho^{2}}\right)} \operatorname{Re}\langle f(t), e\rangle
$$

for a.e. $t \in[a, b]$.

Applying Theorem 2 for $k(t):=\frac{\rho^{2}}{\sqrt{1-\rho^{2}}\left(1+\sqrt{1-\rho^{2}}\right)} \operatorname{Re}\langle f(t), e\rangle$, we deduce the desired result.

In the same spirit, we also have the following corollary.

Corollary 4. If $f \in L([a, b] ; H)$ is such that there exists a vector $e \in H,\|e\|=1$ and $M \geq m>0$ such that either

$$
\operatorname{Re}\langle M e-f(t), f(t)-m e\rangle \geq 0 \text { for a.e. } t \in[a, b],
$$

or, equivalently,

$$
\left\|f(t)-\frac{M+m}{2} e\right\| \leq \frac{1}{2}(M-m) \quad \text { for a.e. } t \in[a, b],
$$


then we have the inequality

$$
\begin{aligned}
& (0 \leq) \int_{a}^{b}\|f(t)\| d t-\left\|\int_{a}^{b} f(t) d t\right\| \\
& \leq \frac{(\sqrt{M}-\sqrt{m})^{2}}{2 \sqrt{m M}} \operatorname{Re}\left\langle\int_{a}^{b} f(t) d t, e\right\rangle \\
& \left(\leq \frac{(\sqrt{M}-\sqrt{m})^{2}}{2 \sqrt{m M}}\left\|\int_{a}^{b} f(t) d t\right\|\right) .
\end{aligned}
$$

The equality holds in (2.15) if and only if

$$
\int_{a}^{b}\|f(t)\| d t \geq \frac{(\sqrt{M}-\sqrt{m})^{2}}{2 \sqrt{m M}} \operatorname{Re}\left\langle\int_{a}^{b} f(t) d t, e\right\rangle
$$

and

$$
\int_{a}^{b} f(t) d t=\left(\int_{a}^{b}\|f(t)\| d t-\frac{(\sqrt{M}-\sqrt{m})^{2}}{2 \sqrt{m M}} \operatorname{Re}\left\langle\int_{a}^{b} f(t) d t, e\right\rangle\right) e .
$$

Proof. The fact that (2.13) and (2.14) are equivalent is a simple exercise and we omit the details.

Observe that (2.13) is clearly equivalent to

$$
\|f(t)\|^{2}+m M \leq(M+m) \operatorname{Re}\langle f(t), e\rangle
$$

for a.e. $t \in[a, b]$, giving the inequality

$$
\frac{\|f(t)\|^{2}}{\sqrt{m M}}+\sqrt{m M} \leq \frac{M+m}{\sqrt{m M}} \operatorname{Re}\langle f(t), e\rangle
$$

for a.e. $t \in[a, b]$.

Since, obviously,

$$
2\|f(t)\| \leq \frac{\|f(t)\|^{2}}{\sqrt{m M}}+\sqrt{m M}
$$

for any $t \in[a, b]$, hence we deduce the inequality

$$
\|f(t)\| \leq \frac{M+m}{\sqrt{m M}} \operatorname{Re}\langle f(t), e\rangle \quad \text { for a.e. } t \in[a, b],
$$

which is clearly equivalent to

$$
\|f(t)\|-\operatorname{Re}\langle f(t), e\rangle \leq \frac{(\sqrt{M}-\sqrt{m})^{2}}{2 \sqrt{m M}} \operatorname{Re}\langle f(t), e\rangle
$$

for a.e. $t \in[a, b]$.

Finally, applying Theorem [2, we obtain the desired result.

Another direction providing different upper bounds for the positive difference

$$
\int_{a}^{b}\|f(t)\| d t-\left\|\int_{a}^{b} f(t) d t\right\|
$$


is outlined in the following.

Corollary 5. If $f \in L([a, b] ; H)$ and $r \in L_{2}([a, b] ; H), e \in H,\|e\|=1$ are such that

$$
\|f(t)-e\| \leq r(t) \quad \text { for a.e. } t \in[a, b],
$$

then we have the inequality

$$
(0 \leq) \int_{a}^{b}\|f(t)\| d t-\left\|\int_{a}^{b} f(t) d t\right\| \leq \frac{1}{2} \int_{a}^{b} r^{2}(t) d t .
$$

The equality holds in (2.17) if and only if

$$
\int_{a}^{b}\|f(t)\| d t \geq \frac{1}{2} \int_{a}^{b} r^{2}(t) d t
$$

and

$$
\int_{a}^{b} f(t) d t=\left(\int_{a}^{b}\|f(t)\| d t-\frac{1}{2} \int_{a}^{b} r^{2}(t) d t\right) e .
$$

Proof. The condition (2.16) is obviously equivalent to

$$
\|f(t)\|^{2}+1 \leq 2 \operatorname{Re}\langle f(t), e\rangle+r^{2}(t)
$$

for a.e. $t \in[a, b]$.

Using the elementary inequality

$$
2\|f(t)\| \leq\|f(t)\|^{2}+1, \quad t \in[a, b],
$$

we deduce

$$
\|f(t)\|-\operatorname{Re}\langle f(t), e\rangle \leq \frac{1}{2} r^{2}(t)
$$

for a.e. $t \in[a, b]$.

Applying Theorem[ 2 for $k(t):=\frac{1}{2} r^{2}(t), t \in[a, b]$, we deduce the desired result.

Finally, we may state and prove the following result as well.

Corollary 6. If $f \in L([a, b] ; H), e \in H,\|e\|=1$ and $M, m:[a, b] \rightarrow[0, \infty)$ with $M \geq m$ a.e. on $[a, b]$, are such that $\frac{(M-m)^{2}}{M+m} \in L[a, b]$ and either

$$
\left\|f(t)-\frac{M(t)+m(t)}{2} e\right\| \leq \frac{1}{2}[M(t)-m(t)] \quad \text { for a.e. } t \in[a, b],
$$

or, equivalently,

$$
\operatorname{Re}\langle M(t) e-f(t), f(t)-m(t) e\rangle \geq 0 \quad \text { for a.e. } t \in[a, b],
$$

hold, then we have the inequality

$$
(0 \leq) \int_{a}^{b}\|f(t)\| d t-\left\|\int_{a}^{b} f(t) d t\right\| \leq \frac{1}{4} \int_{a}^{b} \frac{[M(t)-m(t)]^{2}}{M(t)+m(t)} d t .
$$

The equality holds in (2.20) if and only if

and

$$
\int_{a}^{b}\|f(t)\| d t \geq \frac{1}{4} \int_{a}^{b} \frac{[M(t)-m(t)]^{2}}{M(t)+m(t)} d t
$$

$$
\int_{a}^{b} f(t) d t=\left(\int_{a}^{b}\|f(t)\| d t-\frac{1}{4} \int_{a}^{b} \frac{[M(t)-m(t)]^{2}}{M(t)+m(t)} d t\right) e .
$$


Proof. The condition (2.18) is equivalent to

$$
\begin{aligned}
& \|f(t)\|^{2}+\left(\frac{M(t)+m(t)}{2}\right)^{2} \\
& \leq 2\left(\frac{M(t)+m(t)}{2}\right) \operatorname{Re}\langle f(t), e\rangle+\frac{1}{4}[M(t)-m(t)]^{2}
\end{aligned}
$$

for a.e. $t \in[a, b]$, and since

$$
2\left(\frac{M(t)+m(t)}{2}\right)\|f(t)\| \leq\|f(t)\|^{2}+\left(\frac{M(t)+m(t)}{2}\right)^{2}, \quad t \in[a, b]
$$

hence

$$
\|f(t)\|-\operatorname{Re}\langle f(t), e\rangle \leq \frac{1}{4} \frac{[M(t)-m(t)]^{2}}{M(t)+m(t)}
$$

for a.e. $t \in[a, b]$.

Now, applying Theorem 2 for $k(t):=\frac{1}{4} \frac{[M(t)-m(t)]^{2}}{M(t)+m(t)}, t \in[a, b]$, we deduce the desired inequality.

\section{Additive Reverses for orthonormal Families}

We recall that the family of vectors $\left\{e_{i}\right\}_{i \in\{1, \ldots, n\}}$ in the inner product space $(H ;\langle\cdot, \cdot\rangle)$ is orthonormal if

$$
\left\langle e_{i}, e_{j}\right\rangle=0 \text { if } i \neq j, \quad i, j \in\{1, \ldots, n\} \text { and }\left\|e_{i}\right\|=1 \text { for } i \in\{1, \ldots, n\} .
$$

The following reverse of the continuous triangle inequality for vector valued integrals holds.

Theorem 3. Let $f \in L([a, b] ; H)$, where $H$ is a Hilbert space over the real or complex number field $\mathbb{K},\left\{e_{i}\right\}_{i \in\{1, \ldots, n\}}$ an orthonormal family in $H$ and $M_{i} \in L[a, b]$, $i \in\{1, \ldots, n\}$. If we assume that

$$
\|f(t)\|-\operatorname{Re}\left\langle f(t), e_{i}\right\rangle \leq M_{i}(t) \text { for a.e. } t \in[a, b],
$$

then we have the inequality

$$
\int_{a}^{b}\|f(t)\| d t \leq \frac{1}{\sqrt{n}}\left\|\int_{a}^{b} f(t) d t\right\|+\frac{1}{n} \sum_{i=1}^{n} \int_{a}^{b} M_{i}(t) d t .
$$

The equality holds in 3.2) if and only if

$$
\int_{a}^{b}\|f(t)\| d t \geq \frac{1}{n} \sum_{i=1}^{n} \int_{a}^{b} M_{i}(t) d t
$$

and

$$
\int_{a}^{b} f(t) d t=\left(\int_{a}^{b}\|f(t)\| d t-\frac{1}{n} \sum_{i=1}^{n} \int_{a}^{b} M_{i}(t) d t\right) \sum_{i=1}^{n} e_{i} .
$$

Proof. If we integrate the inequality (3.1) on $[a, b]$, we get

$$
\int_{a}^{b}\|f(t)\| d t \leq \operatorname{Re}\left\langle\int_{a}^{b} f(t) d t, e_{i}\right\rangle+\int_{a}^{b} M_{i}(t) d t
$$


for each $i \in\{1, \ldots, n\}$. Summing these inequalities over $i$ from 1 to $n$, we deduce

$$
\int_{a}^{b}\|f(t)\| d t \leq \frac{1}{n} \operatorname{Re}\left\langle\int_{a}^{b} f(t) d t, \sum_{i=1}^{n} e_{i}\right\rangle+\frac{1}{n} \sum_{i=1}^{n} \int_{a}^{b} M_{i}(t) d t .
$$

By Schwarz's inequality for $\int_{a}^{b} f(t) d t$ and $\sum_{i=1}^{n} e_{i}$, we have

$$
\begin{aligned}
& \operatorname{Re}\left\langle\int_{a}^{b} f(t) d t, \sum_{i=1}^{n} e_{i}\right\rangle \\
& \leq\left|\operatorname{Re}\left\langle\int_{a}^{b} f(t) d t, \sum_{i=1}^{n} e_{i}\right\rangle\right| \leq\left|\left\langle\int_{a}^{b} f(t) d t, \sum_{i=1}^{n} e_{i}\right\rangle\right| \\
& \leq\left\|\int_{a}^{b} f(t) d t\right\|\left\|\sum_{i=1}^{n} e_{i}\right\|=\sqrt{n} \| \int_{a}^{b} f(t) d t \mid
\end{aligned}
$$

since

$$
\left\|\sum_{i=1}^{n} e_{i}\right\|=\sqrt{\left\|\sum_{i=1}^{n} e_{i}\right\|^{2}}=\sqrt{\sum_{i=1}^{n}\left\|e_{i}\right\|^{2}}=\sqrt{n} .
$$

Making use of (3.5) and (3.6), we deduce the desired inequality (3.2).

If (3.3) and (3.4) hold, then

$$
\begin{aligned}
\frac{1}{\sqrt{n}}\left\|\int_{a}^{b} f(t) d t\right\| & =\frac{1}{\sqrt{n}}\left|\int_{a}^{b}\|f(t)\| d t-\frac{1}{n} \sum_{i=1}^{n} \int_{a}^{b} M_{i}(t) d t\right|\left\|\sum_{i=1}^{n} e_{i}\right\| \\
& =\left(\int_{a}^{b}\|f(t)\| d t-\frac{1}{n} \sum_{i=1}^{n} \int_{a}^{b} M_{i}(t) d t\right)
\end{aligned}
$$

and the equality in (3.2) holds true.

Conversely, if the equality holds in (3.2), then, obviously, (3.3) is valid.

Taking into account the argument presented above for the previous result (3.2), it is obvious that, if the equality holds in (3.2), then it must hold in (3.1) for a.e. $t \in[a, b]$ and for each $i \in\{1, \ldots, n\}$ and also the equality must hold in any of the inequalities in (3.6).

It is well known that in Schwarz's inequality $\operatorname{Re}\langle u, v\rangle \leq\|u\|\|v\|$, the equality occurs if and only if $u=\lambda v$ with $\lambda \geq 0$, consequently, the equality holds in all inequalities from (3.6) simultaneously iff there exists a $\mu \geq 0$ with

$$
\mu \sum_{i=1}^{n} e_{i}=\int_{a}^{b} f(t) d t
$$

If we integrate the equality in (3.1) and sum over $i$, we deduce

$$
n \int_{a}^{b} f(t) d t=\operatorname{Re}\left\langle\int_{a}^{b} f(t) d t, \sum_{i=1}^{n} e_{i}\right\rangle+\sum_{i=1}^{n} \int_{a}^{b} M_{i}(t) d t .
$$


Replacing $\int_{a}^{b} f(t) d t$ from (3.7) into (3.8), we deduce

$$
\begin{aligned}
n \int_{a}^{b} f(t) d t & =\mu\left\|\sum_{i=1}^{n} e_{i}\right\|^{2}+\sum_{i=1}^{n} \int_{a}^{b} M_{i}(t) d t \\
& =\mu n+\sum_{i=1}^{n} \int_{a}^{b} M_{i}(t) d t .
\end{aligned}
$$

Finally, we note that (3.7) and (3.9) will produce the required identity (3.4), and the proof is complete.

The following corollaries may be of interest for applications.

Corollary 7. Let $f \in L([a, b] ; H),\left\{e_{i}\right\}_{i \in\{1, \ldots, n\}}$ an orthonormal family in $H$ and $\rho_{i} \in(0,1), i \in\{1, \ldots, n\}$ such that

$$
\left\|f(t)-e_{i}\right\| \leq \rho_{i} \text { for a.e. } t \in[a, b] .
$$

Then we have the inequalities:

$$
\begin{aligned}
& \int_{a}^{b}\|f(t)\| d t \\
& \leq \frac{1}{\sqrt{n}}\left\|\int_{a}^{b} f(t) d t\right\|+\operatorname{Re}\left\langle\int_{a}^{b} f(t) d t, \frac{1}{n} \sum_{i=1}^{n} \frac{\rho_{i}^{2}}{\sqrt{1-\rho_{i}^{2}}\left(1+\sqrt{1-\rho_{i}^{2}}\right)} e_{i}\right\rangle \\
& \leq \frac{1}{\sqrt{n}}\left\|\int_{a}^{b} f(t) d t\right\|\left[1+\left(\frac{1}{n} \sum_{i=1}^{n} \frac{\rho_{i}^{2}}{\sqrt{1-\rho_{i}^{2}}\left(1+\sqrt{1-\rho_{i}^{2}}\right)}\right)^{\frac{1}{2}}\right] .
\end{aligned}
$$

The equality holds in the first inequality in (3.11) if and only if

$$
\int_{a}^{b}\|f(t)\| d t \geq \operatorname{Re}\left\langle\int_{a}^{b} f(t) d t, \frac{1}{n} \sum_{i=1}^{n} \frac{\rho_{i}^{2}}{\sqrt{1-\rho_{i}^{2}}\left(1+\sqrt{1-\rho_{i}^{2}}\right)} e_{i}\right\rangle
$$

and

$$
\begin{aligned}
& \int_{a}^{b} f(t) d t \\
= & \left(\int_{a}^{b}\|f(t)\| d t-\operatorname{Re}\left\langle\int_{a}^{b} f(t) d t, \frac{1}{n} \sum_{i=1}^{n} \frac{\rho_{i}^{2}}{\sqrt{1-\rho_{i}^{2}}\left(1+\sqrt{1-\rho_{i}^{2}}\right)} e_{i}\right\rangle\right) \sum_{i=1}^{n} e_{i} .
\end{aligned}
$$

Proof. As in the proof of Corollary [3 the assumption (3.10) implies

$$
\|f(t)\|-\operatorname{Re}\left\langle f(t), e_{i}\right\rangle \leq \frac{\rho_{i}^{2}}{\sqrt{1-\rho_{i}^{2}}\left(\sqrt{1-\rho_{i}^{2}}+1\right)} \operatorname{Re}\left\langle f(t), e_{i}\right\rangle
$$

for a.e. $t \in[a, b]$ and for each $i \in\{1, \ldots, n\}$.

Now, if we apply Theorem 3 for

$$
M_{i}(t):=\frac{\rho_{i}^{2} \operatorname{Re}\left\langle f(t), e_{i}\right\rangle}{\sqrt{1-\rho_{i}^{2}}\left(\sqrt{1-\rho_{i}^{2}}+1\right)}, \quad i \in\{1, \ldots, n\}, \quad t \in[a, b],
$$

we deduce the first inequality in (3.11). 
By Schwarz's inequality in $H$, we have

$$
\begin{aligned}
& \operatorname{Re}\left\langle\int_{a}^{b} f(t) d t, \frac{1}{n} \sum_{i=1}^{n} \frac{\rho_{i}^{2}}{\sqrt{1-\rho_{i}^{2}}\left(1+\sqrt{1-\rho_{i}^{2}}\right)} e_{i}\right\rangle \\
& \leq\left\|\int_{a}^{b} f(t) d t\right\|\left\|\frac{1}{n} \sum_{i=1}^{n} \frac{\rho_{i}^{2}}{\sqrt{1-\rho_{i}^{2}}\left(1+\sqrt{1-\rho_{i}^{2}}\right)} e_{i}\right\| \\
& =\frac{1}{n}\left\|\int_{a}^{b} f(t) d t\right\|\left(\sum_{i=1}^{n}\left[\frac{\rho_{i}^{2}}{\sqrt{1-\rho_{i}^{2}}\left(1+\sqrt{1-\rho_{i}^{2}}\right)}\right]^{2}\right)^{\frac{1}{2}},
\end{aligned}
$$

which implies the second inequality in (3.11).

The second result is incorporated in:

Corollary 8. Let $f \in L([a, b] ; H),\left\{e_{i}\right\}_{i \in\{1, \ldots, n\}}$ an orthonormal family in $H$ and $M_{i} \geq m_{i}>0$ such that either

$$
\operatorname{Re}\left\langle M_{i} e_{i}-f(t), f(t)-m_{i} e_{i}\right\rangle \geq 0
$$

or, equivalently,

$$
\left\|f(t)-\frac{M_{i}+m_{i}}{2} \cdot e_{i}\right\| \leq \frac{1}{2}\left(M_{i}-m_{i}\right)
$$

for a.e. $t \in[a, b]$ and each $i \in\{1, \ldots, n\}$. Then we have

$$
\begin{aligned}
& \int_{a}^{b}\|f(t)\| d t \\
& \leq \frac{1}{\sqrt{n}}\left\|\int_{a}^{b} f(t) d t\right\|+\operatorname{Re}\left\langle\int_{a}^{b} f(t) d t, \frac{1}{n} \sum_{i=1}^{n} \frac{\left(\sqrt{M_{i}}-\sqrt{m_{i}}\right)^{2}}{2 \sqrt{m_{i} M_{i}}} e_{i}\right\rangle \\
& \leq \frac{1}{\sqrt{n}}\left\|\int_{a}^{b} f(t) d t\right\|\left[1+\left(\frac{1}{n} \sum_{i=1}^{n} \frac{\left(\sqrt{M_{i}}-\sqrt{m_{i}}\right)^{4}}{4 m_{i} M_{i}}\right)^{\frac{1}{2}}\right] .
\end{aligned}
$$

The equality holds in the first inequality in 3.13) if and only if

$$
\int_{a}^{b}\|f(t)\| d t \geq \operatorname{Re}\left\langle\int_{a}^{b} f(t) d t, \frac{1}{n} \sum_{i=1}^{n} \frac{\left(\sqrt{M_{i}}-\sqrt{m_{i}}\right)^{2}}{2 \sqrt{m_{i} M_{i}}} e_{i}\right\rangle
$$

and

$$
\begin{aligned}
\int_{a}^{b} f(t) & d t \\
& =\left(\int_{a}^{b}\|f(t)\| d t-\operatorname{Re}\left\langle\int_{a}^{b} f(t) d t, \frac{1}{n} \sum_{i=1}^{n} \frac{\left(\sqrt{M_{i}}-\sqrt{m_{i}}\right)^{2}}{2 \sqrt{m_{i} M_{i}}} e_{i}\right\rangle\right) \sum_{i=1}^{n} e_{i} .
\end{aligned}
$$

Proof. As in the proof of Corollary 4 from (3.12), we have

$$
\|f(t)\|-\operatorname{Re}\left\langle f(t), e_{i}\right\rangle \leq \frac{\left(\sqrt{M_{i}}-\sqrt{m_{i}}\right)^{2}}{2 \sqrt{m_{i} M_{i}}} \operatorname{Re}\left\langle f(t), e_{i}\right\rangle
$$

for a.e. $t \in[a, b]$ and $i \in\{1, \ldots, n\}$. 
Applying Theorem 3 for

$$
M_{i}(t):=\frac{\left(\sqrt{M_{i}}-\sqrt{m_{i}}\right)^{2}}{2 \sqrt{m_{i} M_{i}}} \operatorname{Re}\left\langle f(t), e_{i}\right\rangle, \quad t \in[a, b], i \in\{1, \ldots, n\},
$$

we deduce the desired result.

In a different direction, we may state the following result as well.

Corollary 9. Let $f \in L([a, b] ; H),\left\{e_{i}\right\}_{i \in\{1, \ldots, n\}}$ an orthonormal family in $H$ and $r_{i} \in L^{2}([a, b]), i \in\{1, \ldots, n\}$ such that

$$
\left\|f(t)-e_{i}\right\| \leq r_{i}(t) \quad \text { for a.e. } t \in[a, b] \text { and } i \in\{1, \ldots, n\} .
$$

Then we have the inequality

$$
\int_{a}^{b}\|f(t)\| d t \leq \frac{1}{\sqrt{n}}\left\|\int_{a}^{b} f(t) d t\right\|+\frac{1}{2 n} \sum_{i=1}^{n}\left(\int_{a}^{b} r_{i}^{2}(t) d t\right) .
$$

The equality holds in 3.14) if and only if

$$
\int_{a}^{b}\|f(t)\| d t \geq \frac{1}{2 n} \sum_{i=1}^{n}\left(\int_{a}^{b} r_{i}^{2}(t) d t\right)
$$

and

$$
\int_{a}^{b} f(t) d t=\left[\int_{a}^{b}\|f(t)\| d t-\frac{1}{n} \sum_{i=1}^{n}\left(\int_{a}^{b} r_{i}^{2}(t) d t\right)\right] \sum_{i=1}^{n} e_{i} .
$$

Proof. As in the proof of Corollary 5 from (2.16), we deduce that

$$
\|f(t)\|-\operatorname{Re}\left\langle f(t), e_{i}\right\rangle \leq \frac{1}{2} r_{i}^{2}(t)
$$

for a.e. $t \in[a, b]$ and $i \in\{1, \ldots, n\}$.

Applying Theorem 3$]$ for

$$
M_{i}(t):=\frac{1}{2} r_{i}^{2}(t), \quad t \in[a, b], i \in\{1, \ldots, n\},
$$

we get the desired result.

Finally, the following result holds.

Corollary 10. Let $f \in L([a, b] ; H),\left\{e_{i}\right\}_{i \in\{1, \ldots, n\}}$ an orthonormal family in $H$, $M_{i}, m_{i}:[a, b] \rightarrow[0, \infty)$ with $M_{i} \geq m_{i}$ a.e. on $[a, b]$ and $\frac{\left(M_{i}-m_{i}\right)^{2}}{M_{i}+m_{i}} \in L[a, b]$, and either

$$
\left\|f(t)-\frac{M_{i}(t)+m_{i}(t)}{2} e_{i}\right\| \leq \frac{1}{2}\left[M_{i}(t)-m_{i}(t)\right]^{2}
$$

or, equivalently,

$$
\operatorname{Re}\left\langle M_{i}(t) e_{i}-f(t), f(t)-m_{i}(t) e_{i}\right\rangle \geq 0
$$

for a.e. $t \in[a, b], \quad i \in\{1, \ldots, n\}$ hold, then we have the inequality

$$
\int_{a}^{b}\|f(t)\| d t \leq \frac{1}{\sqrt{n}}\left\|\int_{a}^{b} f(t) d t\right\|+\frac{1}{4 n} \sum_{i=1}^{n}\left(\int_{a}^{b} \frac{\left[M_{i}(t)-m_{i}(t)\right]^{2}}{M_{i}(t)+m_{i}(t)} d t\right) .
$$


The equality holds in (3.17) if and only if

$$
\int_{a}^{b}\|f(t)\| d t \geq \frac{1}{4 n} \sum_{i=1}^{n}\left(\int_{a}^{b} \frac{\left[M_{i}(t)-m_{i}(t)\right]^{2}}{M_{i}(t)+m_{i}(t)} d t\right)
$$

and

$$
\int_{a}^{b} f(t) d t=\left(\int_{a}^{b}\|f(t)\| d t-\frac{1}{4 n} \sum_{i=1}^{n}\left(\int_{a}^{b} \frac{\left[M_{i}(t)-m_{i}(t)\right]^{2}}{M_{i}(t)+m_{i}(t)} d t\right)\right) \sum_{i=1}^{n} e_{i} .
$$

Proof. As in the proof of Corollary 6] (3.16), implies that

$$
\|f(t)\|-\operatorname{Re}\left\langle f(t), e_{i}\right\rangle \leq \frac{1}{4} \cdot \frac{\left[M_{i}(t)-m_{i}(t)\right]^{2}}{M_{i}(t)+m_{i}(t)}
$$

for a.e. $t \in[a, b]$ and $i \in\{1, \ldots, n\}$.

Applying Theorem 3 for

$$
M_{i}(t):=\frac{1}{4} \cdot \frac{\left[M_{i}(t)-m_{i}(t)\right]^{2}}{M_{i}(t)+m_{i}(t)}, \quad t \in[a, b], \quad i \in\{1, \ldots, n\},
$$

we deduce the desired result.

\section{Applications for Complex-Valued Functions}

Let $e=\alpha+i \beta(\alpha, \beta \in \mathbb{R})$ be a complex number with the property that $|e|=1$, i.e., $\alpha^{2}+\beta^{2}=1$. The following proposition concerning a reverse of the continuous triangle inequality for complex-valued functions may be stated:

Proposition 1. Let $f:[a, b] \rightarrow \mathbb{C}$ be a Lebesgue integrable function with the property that there exists a constant $\rho \in(0,1)$ such that

$$
|f(t)-e| \leq \rho \text { for a.e. } t \in[a, b],
$$

where e has been defined above. Then we have the following reverse of the continuous triangle inequality

$$
\begin{aligned}
& (0 \leq) \int_{a}^{b}|f(t)| d t-\left|\int_{a}^{b} f(t) d t\right| \\
\leq & \frac{\rho^{2}}{\sqrt{1-\rho^{2}}\left(1+\sqrt{1-\rho^{2}}\right)}\left[\alpha \int_{a}^{b} \operatorname{Re} f(t) d t+\beta \int_{a}^{b} \operatorname{Im} f(t) d t\right] .
\end{aligned}
$$

The proof follows by Corollary 3 and the details are omitted.

On the other hand, the following result is perhaps more useful for applications:

Proposition 2. Assume that $f$ and $e$ are as in Proposition [1, If there exists the constants $M \geq m>0$ such that either

$$
\operatorname{Re}[(M e-f(t))(\overline{f(t)}-m \bar{e})] \geq 0
$$

or, equivalently,

$$
\left|f(t)-\frac{M+m}{2} e\right| \leq \frac{1}{2}(M-m)
$$


for a.e. $t \in[a, b]$, holds, then

$$
\begin{aligned}
& (0 \leq) \int_{a}^{b}|f(t)| d t-\left|\int_{a}^{b} f(t) d t\right| \\
\leq & \frac{(\sqrt{M}-\sqrt{m})^{2}}{2 \sqrt{M m}}\left[\alpha \int_{a}^{b} \operatorname{Re} f(t) d t+\beta \int_{a}^{b} \operatorname{Im} f(t) d t\right] .
\end{aligned}
$$

The proof may be done on utilising Corollary 4 but we omit the details

Remark 1. From a practical view point, since

$$
\begin{aligned}
& \operatorname{Re}[(M e-f(t))(\overline{f(t)}-m \bar{e})] \\
= & {[M \alpha-\operatorname{Re} f(t)][\operatorname{Re} f(t)-m \alpha]+[M \beta-\operatorname{Im} f(t)][\operatorname{Im} f(t)-m \beta], }
\end{aligned}
$$

hence a sufficient condition for 4.3 to hold is

$$
m \alpha \leq \operatorname{Re} f(t) \leq M \alpha \text { and } m \beta \leq \operatorname{Im} f(t) \leq M \beta
$$

for a.e. $t \in[a, b]$, where $\alpha, \beta$ are assumed to be positive and satisfying the condition $\alpha^{2}+\beta^{2}=1$. We observe that the above condition (4.6) is very easy to verify in practice, therefore it may useful in various applications where reverses of the continuous triangle inequality are required.

Finally, on making use of Corollary [6] one may state the following result as well:

Proposition 3. Let $f$ be as in Proposition 1 and the measurable functions $K, k$ : $[a, b] \rightarrow[0, \infty)$ with the property that

$$
\frac{(K-k)^{2}}{K+k} \in L[a, b]
$$

and

$$
\alpha k(t) \leq \operatorname{Re} f(t) \leq \alpha K(t) \text { and } \beta k(t) \leq \operatorname{Im} f(t) \leq \beta K(t)
$$

for a.e. $t \in[a, b]$, where $\alpha, \beta$ are assumed to be positive and satisfying the condition $\alpha^{2}+\beta^{2}=1$. Then the following reverse of the continuous triangle inequality is valid:

$$
\begin{aligned}
& (0 \leq) \int_{a}^{b}|f(t)| d t-\left|\int_{a}^{b} f(t) d t\right| \\
\leq & \frac{1}{4} \int_{a}^{b} \frac{[K(t)-k(t)]^{2}}{K(t)+k(t)} d t .
\end{aligned}
$$

The constant $\frac{1}{4}$ is best possible in the sense that it cannot be replaced by a smaller quantity.

Remark 2. One may realise that similar results can be stated if the Corollaries 710 stated above are used. For the sake of brevity, we do not state them here. 


\section{REFERENCES}

[1] J.B. DIAZ and F.T. METCALF, A complementary triangle inequality in Hilbert and Banach spaces, Proceedings Amer. Math. Soc., 17(1) (1966), 88-97.

[2] S.S. DRAGOMIR, Reverses of the continuous triangle inequality for Bochner integral of vector valued function in Hilbert spaces. RGMIA Res. Rep. Coll., to appear.

[3] J. KARAMATA, Teorija i Praksa Stieltjesova Integrala (Serbo-Coratian) (Stieltjes Integral, Theory and Practice), SANU, Posebna izdanja, 154, Beograd, 1949.

[4] M. MARDEN, The Geometry of the Zeros of a Polynomial in a Complex Variable, Amer. Math. Soc. Math. Surveys, 3, New York, 1949.

[5] D.S. MITRINOVIĆ, J.E. PEČARIĆ and A.M. FINK, Classical and New Inequalities in Analysis, Kluwer Academic Publishers, Dordrecht/Boston/London, 1993.

[6] M. PETROVICH, Module d'une somme, L' Ensignement Mathématique, 19 (1917), 53-56.

[7] H.S. WILF, Some applications of the inequality of arithmetic and geometric means to polynomial equations, Proceedings Amer. Math. Soc., 14 (1963), 263-265.

School of Computer Science and Mathematics, Victoria University of Technology, PO Box 14428, MCMC 8001, Victoria, Australia.

E-mail address: sever@csm.vu.edu.au

$U R L:$ http://rgmia.vu.edu.au/SSDragomirWeb.html 